\title{
Surgical Intervention on Uterine Fibromyoma in a Country with Limited Resources: Case of the Gynaecology-Obstetrics Department of the Communal Medical Centre of Ratoma Conakry-Guinea
}

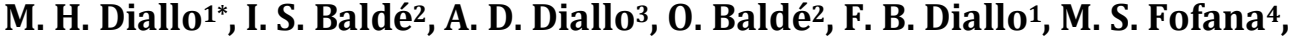 \\ A. Sow ${ }^{2}$, A. B. Barry'2, T. Sy², N. Keita' \\ ${ }^{1}$ Department of Gynaecology-Obstetrics, Donka National Hospital, Conakry, Guinea \\ ${ }^{2}$ Department of Gynecology-Obstetrics, Ignace Deen National Hospital, Conakry, Guinea \\ ${ }^{3}$ Visceral Surgery Department, Donka National Hospital, Conakry Teaching Hospital, Conakry, Guinea \\ ${ }^{4}$ Department of Gynecology-Obstetrics, Ratoma Communal Medical Centre, Conakry, Guinea \\ Email: *hadydiallo2002@yahoo.fr
}

How to cite this paper: Diallo, M.H., Baldé, I.S., Diallo, A.D., Baldé, O., Diallo, F.B., Fofana, M.S., Sow, A., Barry, A.B., Sy, T. and Keita, N. (2020) Surgical Intervention on Uterine Fibromyoma in a Country with Limited Resources: Case of the Gynaecology-Obstetrics Department of the Communal Medical Centre of Ratoma Conakry-Guinea. Open Journal of Obstetrics and Gynecology, 10, 615-625.

https://doi.org/10.4236/ojog.2020.1050055

Received: March 21, 2020

Accepted: April 27, 2020

Published: April 30, 2020

Copyright $\odot 2020$ by author(s) and Scientific Research Publishing Inc. This work is licensed under the Creative Commons Attribution International License (CC BY 4.0).

http://creativecommons.org/licenses/by/4.0/ (c) (i) Open Access

\begin{abstract}
In developing countries, treatment of uterine fibromyoma is confronted with numerous problems, namely: financial inaccessibility to the proposed treatments, fear of surgery and the weakness of the technical platform. Objectives: The objectives of the study were to calculate the frequency of uterine fibromyomas, describe the socio-demographic characteristics of patients, identify the main clinical data and to describe the modalities of surgical management. Patients and Method: It was a mixed descriptive study, cumulative over a period of 5 years (60 months) with data collection in two phases: a 4-year retrospective study from January 1, 2015 to December 31, 2018 and a 1-year prospective study from January 1, 2019 to December 31, 2019. Results: We collected 135 cases of uterine fibromyomas operated on out of a total of 260 cases of gynaecological pathologies, i.e. a frequency of $51.92 \%$. Nulliparous women were the most concerned (45.18\%), and women who attended school (60\%) and those who did not attend school (40\%). Women at home and housewives accounted for $42.20 \%$ and $54.07 \%$ respectively. Clinically, the circumstances of discovery were dominated by menometrorrhagia and menorrhagia respectively $77.77 \%$ and $68.14 \%$. The large uterus was the most frequent physical sign found in $96.29 \%$ of cases. Uterine fibromyomas were recorded in $86.6 \%$ of cases in women with genital activity. The operative in-
\end{abstract}


dications were dominated by the large polymyomatous uterus $(64.44 \%)$, followed by hemorrhagic fibroma $(18.52 \%)$. The surgical treatment was conservative in $92.60 \%$. The total hysterectomy performed in 7.40 . Lethality was $1.4 \%$. Conclusion: The surgical management of fibroids contrasts conservative treatment (myomectomy) with radical treatment (hysterectomy) with multiple possible approaches (hysteroscopy, vaginal surgery, laparoscopy or laparotomy). In our context, only laparotomy was possible due to lack of equipment. Laparoscopy and hysteroscopy equipment is necessary for less invasive surgery.

\section{Keywords}

Fibromyoma, Myomectomy, Hysterectomy, Ratoma

\section{Introduction}

Uterine fibromyomas or myomas or leiomyomas are benign tumors that develop at the expense of the muscle fibers of the uterus. $20 \%-30 \%$ of women over 35 are affected [1]. Myomas affect $20 \%$ - 25\% of women of childbearing age and are 3 - 9 times more common in black women than in white women [2]. The impact of fibromyomas on fertility is controversial, but at date, no study has been able to determine the threshold of number, size, or location of fibroma at which the risk is significant [3].

The exact etiopathogeny of fibroids remains unclear, although many etiological hypotheses are proposed. The hormonal theory is the most logical one, explained by relative or absolute hyperoestrogenicity and the lack of fibroid development after menopause. There is a familial predisposition and factors significantly associated with the growth of fibroids: obesity, under 12 age when first menstruation occurs, nulliparity and infertility [1].

Generally, well tolerated or latent, the variety of anatomical forms would explain the pluralism of clinical expression modes, $50 \%$ of women are asymptomatic and are incidentally discovered during a routine gynecological examination, an ultrasound or pelvic imaging technique. Complementary gynecological means of exploration are ultrasound, hysterosalpingography, hysteroscopy, and magnetic resonance imaging [1].

The clinical symptomatology is dominated by uterine bleeding (menometrorrhagia), pelvic organ compression and painful manifestations [4].

Surgical treatment still remains a very important component and it has benefited in recent years from the contribution of new endoscopic techniques, uterine artery embolization and the treatment with focused ultrasound [1].

In developing countries, treatment is confronted with numerous problems, namely: financial inaccessibility to the proposed treatments, fear of surgery and the weakness of the technical platform [4]. 
Although their degeneration remains insignificant, fibroids are nowadays a real public health problem. In Guinea, the annual statistics of two gynecology-obstetrics departments of the Conakry University Hospital make uterine fibromyomas the first surgical indication for all gynecological pathologies [5]. The high frequency of uterine fibromyomas, difficulties associated with their management, and the inadequacy of previous studies on this pathology in the gynecology-obstetrics department of the Ratoma communal medical center (CMC) motivated the realization of this work.

The objectives of this work were to:

- Calculate the frequency of uterine fibromyomas operated on in the Gynecology-obstetrics department of the CMC in Ratoma.

- Describe the socio-demographic characteristics of the patients concerned.

- Identify the main clinical data of uterine fibroids operated on at the Gynecology-obstetrics Department of the MCC in Ratoma.

- Describe the modalities of surgical management of uterine fibroids in the Gynaecology-obstetrics Department of the CMC in Ratoma.

\section{Patients and Method}

Our study took place in the Gynaecology-Obstetrics department of the Ratoma communal medical centre (a level II facility on the health pyramid of our country).

It was a mixed study of a descriptive and cumulative type carried out over a 5 year period (60 months) with data collection in two phases including a 4 year (60 months ) from January 1, 2015 to December 31, 2018 phase and another 1 year from January 1, 2019 to December 31, 2019 prospective phase.

This study included all patients who underwent uterine fibromyoma surgery during the study period.

Inclusion criteria: We included in this study all patients who had undergone surgery for uterine fibromyoma and had a complete medical record.

Exclusion criteria: Were not included in the study: patients operated on in another facility for uterine fibromyoma and admitted for complicated surgery or other reasons; all patients operated on for conditions other than uterine fibromyoma.

This study was based on a certain number of parameters retained following the review of medical records for the retrospective part and the consultation of patients for the prospective part.

Variables concerned three aspects:

- The epidemiological aspect: the frequency and socio-demographic characteristics which associated age, parity, educational level and socio-professional category.

- The clinical aspect: including the circumstances of discovery (menorrhagia, menometrorrhagia, increase in the volume of the abdomen, infertility test...), 
the data of the clinical and paraclinical examination, associated pathologies, the phase of genital life.

- The therapeutic aspect: grouping together the operative indications, the type of operation performed and the evolution. The collection technique consisted of extracting data from the records for the retrospective part (documentary review), interviewing the patient in bed in hospital and observing patients for the prospective part.

Study population: we carried out an exhaustive census of all cases of uterine fibromyomas operated during the study period at the Gynecology-Obstetrics department of the Ratoma municipal medical center. The results were presented in the form of tables expressed as percentages and averages.

Ethical Consideration: This study protocol was approved by the National Ethics Committee.

\section{Results}

\section{Frequency}

During the study period, we collected 135 cases of uterine fibromyomas operated on out of a total of 260 cases of gynecological pathologies operated on at the Gynecology-Obstetrics Department of the MCC in Ratoma, i.e. a frequency of $51.92 \%$.

The highest case frequencies were observed in 2015 (28.88\%), 2016 (19.26\%) and in 2017 (24.45\%) (Table 1).

\section{Socio-Demographic Characteristics}

Age: The average age of our patients was 37 with extremes of 20 and 54. The age group most affected by UF was that of 30 - 39 with a frequency of $49.63 \%$. The frequency was identical for the $20-29$ and $40-49$ age groups (23.70\%). Those aged 50 and over were less affected (2.97\%).

Parity: nulliparous were the most concerned $(45.18 \%)$, followed by primiparous $(24.44 \%)$, pauciparous represented $(15.60 \%)$ followed by multiparous (13.33\%) and major multiparous (1.48\%).

Educational level: the analysis of this parameter shows a higher frequency among educated women (60\%); however, this rate is not negligible among uneducated women (40\%).

Socio-professional category: housewives were the most affected (42.20\%), followed by women in liberal professions and salaried women with $32.60 \%$ and $20.00 \%$ respectively. Pupils and students accounted for $5.20 \%$.

Marital status: married women were the most affected (54.07\%), single women also represented a considerable proportion (4.0\%).

\section{Clinical Features}

The circumstances of discovery were dominated by the menometrorrhagia (77.77\%) followed by menorrhagia (68.14\%), a sensation of abdominopelvic mass (35.55\%) and infertility assessment (10.37\%). One patient had 2 or more functional signs (Table 2). 
Table 1. Annual frequency of UF performed at Ratoma MCC.

\begin{tabular}{ccc}
\hline Year & Number of UF operated & Percentage (\%) \\
\hline 2015 & 39 & 28.88 \\
2016 & 26 & 19.26 \\
2017 & 33 & 24.45 \\
2018 & 15 & 11.11 \\
2019 & 22 & 16.30 \\
Total & 135 & 100 \\
\hline
\end{tabular}

Table 2. Distribution of patients by circumstances of discovery.

\begin{tabular}{ccc}
\hline Circumstances of Discovery & $\mathbf{n}=\mathbf{1 3 5}$ & Percentage (\%) \\
\hline menometrorrhagia & 106 & 77.77 \\
Menorrhagia & 92 & 68.14 \\
Feeling of abdominopelvic mass & 48 & 35.55 \\
Infertility check-up & 14 & 10.37 \\
Fortuitous discovery & 13 & 9.62 \\
Pelvialgies & 11 & 8.14 \\
Increase in the volume of the abdomen & 9 & 6.67 \\
Pollakiurie & 7 & 5.18 \\
Miscarriages & 5 & 3.70 \\
\hline
\end{tabular}

The large uterus $(96.29 \%)$ and genital hemorrhage (72.59\%) were the most common signs (Table 3).

Only one patient had 2 or 3 functional signs.

Ultrasound was performed in most cases $(98.51 \%)$ and hysterosalpingography more rarely (5.18\%) (Table 4).

Type 0 of the FIGO classification was the most encountered (29.62\%) followed by type $2(25.18 \%)$ and $3(20.75 \%)$ (Table 5 ).

\section{Existence of a gynecological pathology associated with ultrasonography}

Based on the ultrasound examination, the fibroid was associated with gynaecological pathology in $11.1 \%$ of cases. It was an ovarian cyst in 12 cases $(8.88 \%)$ and hydrosalpinx in 3 cases (2.22\%).

Genital life phase: Uterine fibromyoma was recorded in $86.6 \%$ of cases in women during genital activity, in $10.37 \%$ in perimenopausal women and in $2.97 \%$ in postmenopausal women.

\section{Surgical management}

\section{Patient Distribution by Surgery Indication}

Surgical indications were dominated by the large polymyomatous uterus (64.44\%), followed by the hemorrhagic fibroma (18.52\%) and infertility which accounted for $17.04 \%$.

Distribution of patients according to the surgical procedure performed. 
Table 3. Patients distribution according to gynecological examination data.

\begin{tabular}{ccc}
\hline Gynaecological examination data & $\mathbf{n}=\mathbf{1 3 5}$ & Percentage (\%) \\
\hline Large uterus & 130 & 96.29 \\
Genital Hemorragia & 98 & 72.59 \\
Apparently Normal & 5 & 3.70 \\
Polyp delivered through the cervix & 14 & 10.37 \\
Exo cervicitis & 12 & 8.88 \\
Adnexal mass & 7 & 5.18 \\
\hline
\end{tabular}

Table 4. Distribution of patients according to whether or not additional diagnostic tests were performed.

\begin{tabular}{ccc}
\hline Additional diagnostic tests & $\mathbf{n}=135$ & Percentage \\
\hline Abdominopelvic ultrasound & 133 & 98.51 \\
Hysterosalpingography & 7 & 5.18 \\
Nothing & 2 & 1.48 \\
\hline
\end{tabular}

Table 5. Distribution of patients by topographic shape of fibromyomas on ultrasound (FIGO Classification).

\begin{tabular}{ccc}
\hline FIGO Classification & $\mathbf{n}=\mathbf{1 3 5}$ & Percentage (\%). \\
\hline Type 0 & 40 & 29.62 \\
Type 1 & 28 & 20.74 \\
Type 2 & 34 & 25.18 \\
Type 3 & 29 & 20.75 \\
Type 4 & 17 & 12.59 \\
Type 5 & 18 & 13.33 \\
Type 6 & 16 & 11.85 \\
Type 7 & 13 & 9.62 \\
Type 8 & 14 & 10.37 \\
\hline
\end{tabular}

The surgical treatment was conservative in 125 cases $(92.60 \%)$ and consisted of a simple myomectomy and/or polymyomectomy. The whole hysterectomy was performed in 10 cases $(7.40 \%)$. Associated lesions were also managed simultaneously (cystectomy, hydrosalpinx cure).

\section{Results of the anatomopathological examination}

Out the 135 surgeries performed for uterine fibromyoma, 80 pieces (59.25\%) were examined with confirmation of the diagnosis of leiomyoma in all cases, while uterine fibromyoma was associated with endometrial hyperplasia in 7 cases of hysterectomy (5.18\%), an ovarian cyst in 12 cases $(8.88 \%)$ and a cervical cancer in one extrafacial hysterectomy case $(0.74 \%)$.

\section{Prognosis}

Per and postoperative complications 
Intraoperative complications were dominated by haemorrhage, which affected 35 patients $(25.92 \%)$, with $65.18 \%$ of post-operative complications being uncomplicated and $34.82 \%$ complicated. These complications were dominated by anaemia (haemoglobin level lower than $10 \mathrm{~g} / \mathrm{dl}$ ) in 32 patients (23.70\%) whose myomectomy was haemorrhagic and required a blood transfusion in 21 cases (27.15\%).

We recorded 2 death cases due to severe anemia complicated by hypovolemic shock, i.e. a case-fatality rate of $1.48 \%$.

\section{Discussion}

\section{Frequency}

The highest frequencies of cases observed in 2015, 2016 and in 2017 are linked to the closure of the Donka National Hospital for renovation, resulting in a considerable increase in consultations at the Ratoma municipal medical center.

Our frequency is higher than those reported by some authors, notably: Mahbouli, S. et al. [6], in Tunisia reporting a rate of $19.1 \%$ in relation to all gynaecological surgery and Laghzaoui, M. et al. [7] in Morocco reporting a rate of 15\% in relation to all gynaecological pathology. On the other hand, this frequency is lower than those reported by other authors, notably Dia, A. et al. [8] who reported a rate of $58.62 \%$ of all gynaecological disorders operated on. SY, T. et al. [5] in Guinea reported a frequency of $49.92 \%$. Baldé, I. S. et al. [4] reported a frequency of $42.4 \%$ at the Conakry University Hospital.

Our high frequency could be explained by the fact that the study took place in a level II reference centre in the health pyramid of our country, which is easily accessible and the cost of the intervention affordable. This frequency is often underestimated because it only takes into account operated uterine fibromyomas which are symptomatic as described by Cramer and Patel (quoted by Razafindrabe, J. A. [9]). On a serial anatomopathological study of 100 consecutive hysterectomies for usual pathologies, the incidence of uterine fibromyomas rose from $33 \%$ of preoperative diagnosis to $77 \%$ after an anatomopathological study (less than $1 \mathrm{~cm}$ ).

\section{Socio-demographic characteristics}

Concerning age, our result is different from those described in the literature: 43 years according to Parazzini quoted by Mahbouli [6]; 40 - 49 years according to Dia et al. [8], in Senegal, i.e. $43.6 \%$. In our series, a rate of $23.70 \%$ was recorded for the 20 - 29 age group, which reinforces the idea that in black women, uterine fibromyomas are increasingly found in young people.

Regarding parity, our result is different from that of some authors, notably Mahbouli, S. et al. [6] in Tunisia who report similar rates between nulliparous (30.6\%), pauciparous (31.5\%) and multiparous (37.9\%).

Baldé, I. S. et al. [4] in Guinea reported a rate of $32.8 \%$ for nulliparous and $23.8 \%$ for pauciparous. This difference could be explained by the difference between the physio-anatomical data of black women and those from the Maghreb. 
However, the notion reported by ROSS et al. [10] that the relative risk of having a uterine fibromyoma decreases with parity is not unanimous. The high frequency in nulliparous women could be explained by the relationship between infertility and uterine fibromyomas, due to the climate of hyperoestrogenism which could be at the root of this phenomenon which has a socio-cultural importance especially in developing countries where societies advocate pro-natalist behaviour.

With regard to the level of education, our findings are consistent with those of Baldé, I. S. et al. [4] in Guinea, who reported a rate of $54.8 \%$ among educated women and $45.2 \%$ among unschooled women, respectively. There is no proven relationship between the occurrence of uterine fibromyoma and a woman's level of education. However, educated women have more access to health care than uneducated women, they use contraceptives and often marry late, so they are more exposed to the effects of estrogen. The high frequency of housewives in our series could be explained by the fact that they make up the bulk of the female population in Guinea [11].

\section{Clinical Features}

Our results are similar to those of Laghzaoui, M. et al. [6] in Morocco reporting that the functional signs of uterine fibromyomas were dominated by genital haemorrhages (68.26\%) and these haemorrhages were represented essentially by menorrhages (68.3\% of cases). On the other hand, Dia, A. et al. [8] in Senegal reported that the increase in the volume of the abdomen was the most frequent reason for consultation ( $96.4 \%$ of cases). This difference could be explained by the difference in vocation between the study services (Surgery in Senegal and Gynecology-Obstetrics in Senegal).

Patients with an abdominopelvic mass are more likely to use Surgery than Gynecology services and conversely for those with genital bleeding. The predominance of large uterus and genital haemorrhage has also been reported in the Baldé, I. S. [4] and Razanfindrabe, J. A. et al. [9] studies. The same is true for pathologies associated with uterine fibromyoma.

Additional diagnostic tests were performed in $98.51 \%$ of cases. Among these patients all benefited from abdominal pelvic ultrasound, first-line examination and 7 benefited from hysterosalpingography (5.18\%). Magnetic resonance imaging and hysteroscopy were not performed due to the lack of equipment. Dia, A. et al. [8] in Senegal reported that abdominopelvic ultrasound was performed in $42.28 \%$ of cases and hysterosalpingography in $12.14 \%$.

Contrary to Lagzaoui, M. et al. [7] reporting the realization in $100 \%$ of the cases in his series. The closer one is to menopause the more the number of uterine fibromyomas decreases, the same observations were made in the study of Mahbouli, S. et al. [6] in Tunisia reporting in their series 75\% of women in genital activity period; $14.6 \%$ in the perimenopausal period and $10 \%$ of women in confirmed menopause in whom the age of menopause appears to be delayed (51.25 years). 
With regard to the existence of a gynaecological pathology associated with the fibroid, our observation is related to Célui de Laghzaoui, M. et al. [7] in Morocco who reported in his series that according to ultrasound, uterine fibroid was associated in 24 cases with a cyst of the ovary and a hydrosalpinx in 17 cases.

\section{Surgical management}

Concerning the operative indication, our observation is shared by Langzaoui, M. et al. [7] in Morocco, reporting in his series that the operative indications were dominated by large uterine fibroids $(41 \%)$ and large polymyomatous uteri (38.16\%). Therapeutic intervention must be motivated by the existence of an embarrassing symptom and only clinical surveillance is necessary in the case of asymptomatic fibroma. A possible future desire for pregnancy must be taken into account as well as the age of occurrence of menopause [12].

The choice of myomectomy and hysterectomy was guided by several factors, of which age and parity were the most important. The size of the uterus and anatomical features were rather decisive in the choice of the surgical approach. Myomectomy was suggested for young women and those who wish to become pregnant in the absence of pregnancy. This choice is still not possible, especially when there are many fibroids, and there is a risk that after myomectomy a fibrous stunted uterus may be left behind, sometimes with tubo-ovarian adhesion sclerosis, which may compromise reproductive function and which is not safe from recurrence.

Our finding is different from that of Dia, A. et al. [8] in Senegal, who reported more hysterectomies in his series: $35.72 \%$ subtotal and $27.86 \%$ total hysterectomy, with a myomectomy rate performed on $43 \%$ of cases in his series, performed by three routes: abdominal (80.8\%), hysteroscopic (13.8\%) and laparoscopic (5.3\%). We did not perform hysteroscopy and laparoscopy due to the lack of equipment.

\section{Prognosis}

Anaemia was found in the study by Mahbouli, S. et al. [6] in Tunisia reporting $54 \%$ with a haemoglobin level under $12 \mathrm{~g} / \mathrm{dl}$. Baldé, I. S. et al. [4] also found anaemia in their series. Dia, A. et al. in Senegal reported $0.7 \%$ deaths, while Mahbouli et al. [6] in Tunisia reported no deaths in their series of 219 patients.

Data in the literature reported a lethality lower than $1 \%$ [13] [14].

Our high lethality rate $(1.48 \%)$ is thought to be related to the lack of resuscitation equipment.

\section{Limitations and difficulties of the study}

Encountered problems were:

- Incomplete records.

- Inadequacy in the summary description of results: in particular the precision of the location and ultrasound characteristics of the myomas observed.

- The lack of equipment for hysteroscopy.

\section{Conclusions}

The frequency of uterine fibroids is important in gynaecological surgery; signs of 
discovery of myomas that may point to a surgical solution may be menorrhagia, metrorrhagia or menometrorrhagia most often leading to iron deficiency anaemia, pelvic pain or heaviness, compression of neighbouring organs (urinary, digestive or vascular). The surgical management of fibroids opposes the conservative treatment (myomectomy) with the radical treatment (hysterectomy) with multiple possible approaches (hysteroscopy, vaginal surgery, laparoscopy or laparotomy).

In our context only laparotomy was possible due to a lack of equipment. The surgical indications vary from one patient to another and must take into account the desire for fertility, the psychology of the patient, the surgical risks and the medium and long-term consequences of the operation. It is important, in the context of myomatous pathology, to surgically treat only fibroids that are symptomatic or may affect fertility.

The objectives of surgical treatment are multiple, namely the improvement of symptoms caused by fibroids, the improvement of fertility, and in some cases the prevention of serious complications inherent to fibroids. The surgical treatment of fibroids is presented as a curative solution as opposed to medical treatment, which is usually only symptomatic. Surgical proposals must always integrate patients' wishes, such as the desire for pregnancy, the desire to preserve the uterus or the ovarian function (pre-menopause, peri-menopause, menopause).

\section{Conflicts of Interest}

The authors declare no conflicts of interest regarding the publication of this paper.

\section{References}

[1] National College of French Gynecologists and Obstetricians (CNGOF) (2011) Abstract of Gynecology-Obstetrics. 2nd Edition, Masson, Paris, 227-228.

[2] Okogbo, F.O., Ezechi, O.C., Loto, O.M. and Ezeobi, P.M. (2011) Uterine Leimyomatan in South Western Nigeria: A Clinicalstudy of Presentations and Management Outcome. African Health Sciences, 11, 271-278.

[3] Bajekal, N. and Li, T.C. (2000) Fibroids, Infertility and Pregnancy Wastage. Human Reproduction Update, 6, 614-20. https://doi.org/10.1093/humupd/6.6.614

[4] Baldé, I.S., Diallo, B.S., Conté, I., Baldé, O., Diallo, M.H., Diallo, B.C., Mamy, M.N., Sy, T. and Keita, N. (2015) Uterine Fibromyomas: Epidemiological and Clinical Aspects, Surgical Management and Prognosis at Conakry University Hospital. Revue Internationale des Sciences Médicales, 17, 118-124.

[5] Sy, T., Diallo, Y., Diallo, A.B., Diallo, F.B., Touré, A., Keita, N. and Diallo, M.S. (2007) Uterine Fibromyomas: Epidemioclinical Aspect and Surgical Management at the Ignace Deen Gynecology-Obstetrics Clinic of the CHU of Conakry. Annals of the University of Ouagadougou, 5, 113-126.

[6] Mahbouli, S., Messaoudi, Y., Chandoul, Y., Zayene, H., Messaoudi, F., Basly, M. and Messaoudi, L. (2001) Management of Uterine Fibroids (About 219 Cases). Tunisie Médicale, 79, 515-520.

[7] Laghzaoui, M., Boukaidi, S., Bouhya, S., Hermas, S., Bennani, O. and Aderdour, M. 
(2001) Epidemiology of Uterine Fibroids (About 690 Cases). Maroc Medical, 23, 266-270.

[8] Dia, A., Beye, S.B., Dangou, J.M., Dieng, M. and Woto Gaye, C.T. (2003) Uterine Fibroids at the Dakar University Clinic: About 140 Cases Operated on in Two Years. Dakar Médical, 48, 72-76.

[9] Razafindrabe, J.A., Rabarijaona, M., Rakotoarisoa, B., Radriamaro, B., Sambany, R. and Razafindramboah (2002) Reasons for Hysterectomy in a Gynecology and Obstetrics Department. Medicine d'Afrique Noire, 49, 166-168.

[10] Ross, R.K., Pike, M.C. and Vessey, M.P. (1986) Risk Factors for Uterine Fibroids: Reduced Risk Associated with Oral Contraceptives. British Medical Journal, 293, 359-362. https://doi.org/10.1136/bmj.293.6543.359

[11] Diallo, M.D.D. and Bussangu, M.F. (2012) Archive of the Ministry of Planning, National Institute of Statistics, Conakry, Guinea: Demographic and Health Survey (DHS). INS and ORC Marco, Calvelton, Maryland, USA, 246-247.

[12] Bezard, F. (2001) Uterine Fibromyoma: Treatment. Drug Treatments for Uterine Fibroma. Argumentaire, 14, 433-445.

[13] Abbara, A. (2004) Uterine Fibromyoma: Generalities. Journal de Gynécologie Obstétrique et Biologie de la Reproduction, 42, 716-719.

[14] Zhioua, F., Ferciou, M., Mouelhi, C., et al. (1997) Contribution of Operative Hysteroscopy in the Treatment of Intrauterine Myomas in Infertile Patients. Maghreb Medical, 320, 34-36. 